\title{
Some Results on the Evolution of Primordial Black Holes
}

\author{
J. E. Horvath and P. S. Custódio \\ Instituto de Astronomia, Geofísica e Ciências Atmosféricas \\ Rua do Matão 1226, 05508-900 \\ São Paulo SP, Brazil \\ (Received on 15 October, 2005)
}

\begin{abstract}
We briefly review some recent results related to thermodynamics of semiclassical black holes applied to their primordial formation. Issues on the existence of an influx of ambient particles onto the PBHs, which may help grow them, are addressed. We revisit the integrated flux from evaporated PBHs contributing to the present backgrounds and show that there probe mass scales which are otherwise poorly constrained. Finally the Generalized Second Law of Thermodynamics and the Holographic Principle are combined to show that if some form of the latter holds, strong upper bounds to the mass density of PBHs formed in the early universe may be obtained, especially for inflationary cosmological models. This method is completely independent from those based on the background fluxes and applies to potentially important epochs of PBH formation, resulting in quite strong constraints to $\Omega_{p b h}$.
\end{abstract}

\section{INTRODUCTION}

Small primordial black holes (PBHs) formed in some stage(s) of the early universe are certainly not very abundant today [1-4]because these objects evaporate quickly due to Hawking radiation [1]. A review of their formation and limits has been recently given in Ref.[5], showing the constraints for several initial PBH masses and formation scenarios.

Generally speaking, most of the methods to constrain the abundance of PBHs are based on comparisons of the integrated contribution of Hawking radiation with some background flux. Related methods such as deuterium abundance analysis and ${ }^{4} \mathrm{He}$ spallation have been also employed to extend the bounds. Although the figures depend on the considered interval of masses, it is fairly general to say that present limits suggest $\Omega_{p b h}<10^{-6}$ for PBHs with masses $\leq$ the Hawking mass $M_{\text {haw }} \equiv 10^{15} \mathrm{~g}$ (defined as the mass-scale which is evaporating precisely today, within a small numerical factor).

\section{HAWKING EMISSION AND ACCRETION OF PBHS SOME NEW RESULTS}

The celebrated result by Hawking [1], that black holes would loss their mass through an irreversible quantum process allowed a definition of a temperature for black holes and brought these objects to the realm of Thermodynamics. This energy loss process gives rise to a continuous increase of the temperature. It has been shown that the emitted spectrum is thermal, with an associated temperature (for Schwarzchild black holes),

$$
T_{b h}=\frac{\hbar c^{3}}{8 \pi G k_{B} M} \sim \frac{10^{-7} K}{\left(M / M_{\odot}\right)} .
$$

assuming the validity of Stefan-Boltzmann law and the formula above, the evolution of the mass of the $\mathrm{PBH}$ is given by

$$
\frac{d M}{d t}=-\frac{A(M)}{M^{2}}
$$

where $A(M)$ counts the degrees of freedom of the emitted particles. (see [6] for details).

Since we know that the cosmological environment was very hot and dense in the radiation era, therefore, we expect some classical absorption of the energy-matter from the surroundings of a given $\mathrm{PBH}$ if its associated temperature at formation happens to be lower than the cosmic temperature. for example, in the radiation-dominated era the mass inside the horizon is

$$
M_{h o r}(t) \sim 7.6 \times 10^{37}(t / 1 s) g
$$

This relation is very important to the problem, since we expect that PBHs would be formed with masses comparable to the horizon mass [2]. Since PBHs with initial masses larger than the horizon mass are excluded by causality (but see Harada and Carr [7]), a generic PBH with initial mass $M$ formed in the early universe its formation time must satisfy

$$
t_{F}(M) \geq 3 \times 10^{-38}(M / g) s .
$$

Therefore, from $t_{F}(M)$, we expect that the PBH mass will be below the horizon mass at subsequent times $t>t_{F}$.

To address the effects of the environment on the evolution of PBHs masses, we proceed to include a classical absorption term. This term can be constructed as $\left(\frac{d M}{d t}\right)_{a b s}=$ $\sigma_{g}(M) F_{\text {rad }}(T)$ where $\sigma_{g}(M)=\frac{27 \pi}{4} r_{g}^{2}$ is the gravitational cross section for the capture of relativistic particles [8]. Since $F_{\text {rad }}(T)=c \rho_{\text {rad }}(T)$ is the radiative flux as seen in the rest frame of the black hole, the complete differential equation for the mass is

$$
\frac{d M}{d t}=-\frac{A(M)}{M^{2}}+\frac{27 \pi G^{2}}{c^{3}} \rho_{r a d}(T) M^{2} ;
$$

where we have assumed that $M>M_{\text {Planck }}$ in order to avoid the issue of the final evaporation stage. Corrections due to quantum gravity effects and so-called "grey" factors have been neglected, as well as the back-reaction (see [9] and references therein for a quantum mechanical evaluation). 


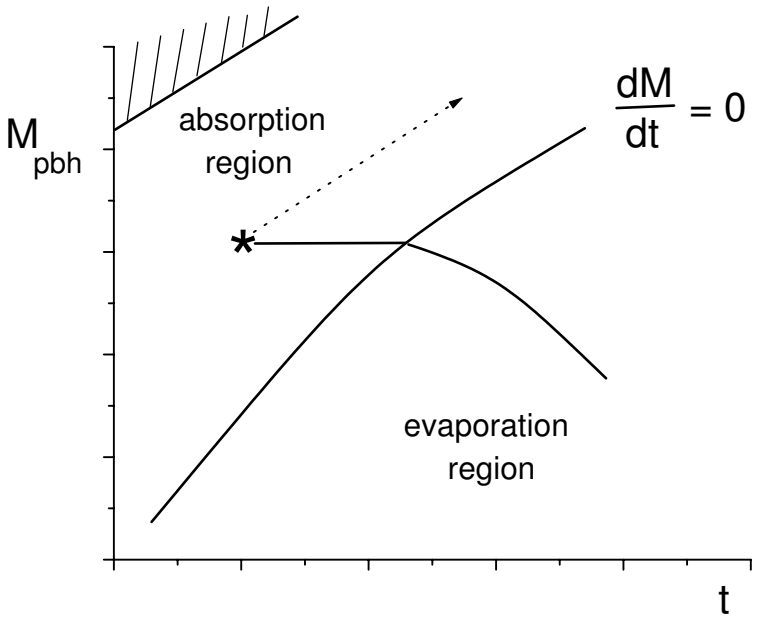

FIG. 1: Primordial black holes

It can be checked that PBHs formed obeying the causality constraints with masses $\geq M_{\text {Haw }}$ before $t \sim 1 s$ will experience an inward heat flux from the hotter surroundings, gaining mass-energy at the expense of the radiation. This gain must stop when the temperature of these PBHs equals to radiation temperature, achieving instantaneous equilibrium and $\dot{M}=0$ at this instant. We can calculate the locus of the equilibrium mass at any epoch solving $\dot{M}=0$ [4], yielding

$$
M_{c}(t) \sim \frac{10^{26} g}{\left(T / T_{0}\right)}
$$

where $T_{0}$ is the present cosmic temperature. Any $\mathrm{PBH}$ with mass greater than this value at $t$ must be accreting $(\dot{M}(M>$ $\left.M_{c}\right)>0$ ) and not evaporating, since the Hawking evaporation is negligible in this case.

Assuming that PBHs are not important for the overall expansion, we can quickly solve the problem of $\mathrm{PBH}$ growth epoch by inserting the appropriated cosmological temperature and density and solving for $M(t)$. The results are summarized as follows:

a) the solution are never of a runaway type [8], instead it is found that the growth of the PBHs mass is small (less than a few percent) in the radiation-dominated era.

b) in spite of the above, accretion is important for PBHs because it delays the beginning of the evaporation era (see Fig. 1)

c) PBHs growing substantially by accreting quintessence fields [10] are fine-tuned in Newtonian gravity [11], and vanish entirely in a fully relativistic treatment [12].

\section{LIMITS TO PBH ABUNDANCE USING THE KNOWN RADIATION BACKGROUNDS}

It is possible to constrain the mass abundance of unclustered PBHs formed with a single mass and subject to the
Hawking evaporation and particle absorption from the environment by demanding their emission to be lower than the observed backgrounds. Since the radiative flux is proportional to the numerical density, an upper bound is obtained by comparing the calculated and observed diffuse background values, for finite bandwidths. For a wide range of formation redshifts, we found [13] that the bounds are better than several values obtained by other arguments $\Omega_{p b h} \leq 10^{-10}$; and they apply to PBHs which are evaporating today.

Taking into account the radiation at the sources, and transforming to the physical frame, an evaluation the radiation received today $(z=0)$ from all these PBHs yields

$$
\delta F_{p b h}=\frac{3 L_{0} M_{H a w} \xi_{0}}{4 \pi} \int_{\varepsilon_{\odot}}^{z_{i n i}} d z \frac{\left[\mu_{*} F\left(\mu_{*}, z\right)\right]^{-2}}{D_{L}^{2}(z)(1+z)}
$$

which may be compared with the observed backgrounds. The argument applies to all PBHs which evaporate, since their radiation must appear at some wavelength depending on the formation epoch. Since the present-day critical mass is $M_{C 0} \sim 10^{26} g \sim 10^{-7} M_{\odot}$, this kind of method can probe mass scales between the Hawking mass and $M_{C 0}$, which may go unnoticed by other methods. See [13] for a detailed account of this method and results.

\section{ENTROPY BOUNDS AND THE GLS: STRONG LIMITS TO PBH ABUNDANCE?}

It has been a lot of recent work $[14,15]$ about the possibility of gravity being "holographic", in the sense that the gravitational field may be completely specified by the values at the boundaries of the spacetime containing it. This ideas are tied to the formulation of the so-called Holographic Principle, which states that for a given volume $V$, the state of maximal entropy is proportional to the area $A$ bounded by the volume $V$. The microscopic entropy $S$ associated with the volume $V$ is bounded absolutely by the Bekenstein-Hawking value $S_{B H} \leq \frac{A}{4}$, in which the Planck length $L_{p}$ has been set to unity.

A second important concept to be used below is the generalized Second Law of thermodynamics, first formulated by Bekenstein in 70s [16]. Bekenstein noted that as black holes absorb matter, the entropy of the universe seemed to decrease, since the matter entropy vanished behind the horizon. This was quite problematic, since this irreversible $G e$ roch process seems to go against the second law of thermodynamics $\Delta S_{\text {matter }}>0$.

In order to recover the second law of thermodynamics, Bekenstein [16]conjectured a Generalized Second Law of Thermodynamics (or GSL), adding to the consideration the black hole entropies (which are proportional to their horizon areas). Then, for $N$ black holes + radiation and matter, the GSL takes the form

$$
S_{\text {total }}=S_{m-r}+\frac{1}{4} \sum_{i}^{N} A_{i}
$$




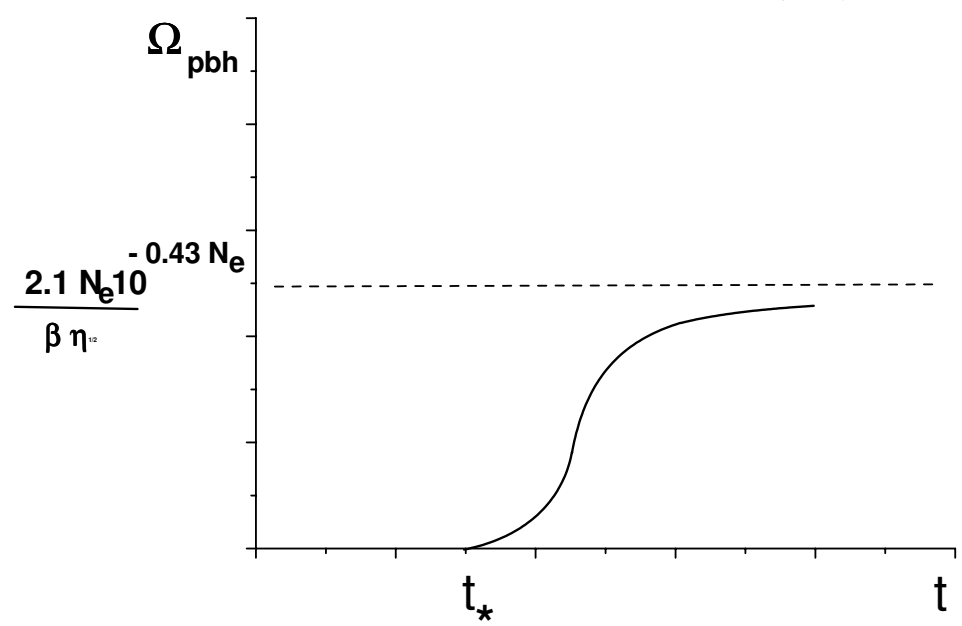

FIG. 2: Primordial black hole abundance limits within inflationary models. As discussed in the text, $\Omega_{p b h} \equiv 0$ before $t_{*}$. Note that even for PBHs formed at asymptotic times (that is, when $G \rightarrow 1$ ) the allowed contribution to $\Omega_{p b h}$ is very small because of the suppression by $N_{e}$. Physically the suppression reflects the fact that the volume grows much more than the entropy inside the particle horizon.

As long as we deal with classical process involving black holes and matter, the total variation of entropy must be positive $\Delta S_{\text {total }}>0$.

The GSL can be used analogously to the well-known use of the second law for ordinary systems. We used eq.() together the GSL for a given box with a well-defined energy and radius to show how to obtain upper limits to the black hole masses formed inside the box. Later we extended the analysis including the Holographic Bound, $S_{\text {total }}<\frac{A}{4 L_{p l}^{2}}$, conjectured to be valid for strongly gravitating systems, to repeat the exercise and show the limits for the case of realistic cosmologies.

The formation of PBHs inside an inflationary era dilutes the abundance of these objects much in the same way as the dilution of monopoles or any other relict, and is of no practical interest. However, the surviving PBHs abundances after the end of an inflationary era (assuming that inflation ends with a reheating phase)puts interesting limits onto $\Omega_{p b h}$.

We have evaluated the maximal PBH abundances at $t>t_{f}$ as follows. We assumed that the inflationary phase lasts $\Delta t \leq$ $t_{f}$, and from $t_{f}=\Gamma \times 10^{-35} s$ on (with $1<\Gamma<10^{3}$ being a model-dependent parameter including the unknown details of the epoch) the universe entered the radiation-dominated era. The numerical details of this transition are not important, as long as it occurs immediately after $t_{f}$. The continuity of the particle horizon allows us write $R_{p h}(t)=\frac{c t_{f}}{(1-n)}\left(t / t_{f}\right)$. This value is adopted as the initial $R_{i}$ of the radiation-dominated era $R_{p h}\left(t_{f}\right) \equiv R_{i}=\frac{c t_{f}}{(1-n)}=L_{p l} \exp \left(N_{e}\right)$.

Therefore, $R_{p h}(t) \sim L_{p l} \exp \left(N_{e}\right)\left(t / t_{f}\right)$ for $t>t_{f}$, and the available entropy evolves as

$$
S_{p h}(t)=\pi \exp \left(2 N_{e}\right)\left(t / t_{f}\right)^{2}
$$

Demanding that there must be enough entropy to create the black holes inside the particle horizon, we can proceed to evaluate the maximal abundances in PBHs formed at a time $t_{F}$. Most of the models for $\mathrm{PBH}$ formation indicate that the $\mathrm{PBH}$ mass at its formation, $t_{F}$, is a fraction $\beta \leq 1$ of the particle horizon. The result is

$$
\Omega_{p b h}\left(N_{e}, t_{F}\right)<\frac{2 \times N_{e}}{\beta \eta^{1 / 2}} \times G\left(N_{e}, t_{F}\right) \times 10^{-\frac{43}{100} N_{e}}
$$

with

$$
G\left(N_{e}, t\right)=1-\frac{S_{\text {rad }}}{\pi\left(t / t_{f}\right)^{2}} \exp \left(-2 N_{e}\right)
$$

The PBH abundance is zero when the function $G\left(N_{e}, t_{F}\right)$ vanishes, this happens at a certain value $t_{*} \sim 0.9 N_{e} \eta^{-1 / 2} \exp \left(-N_{e}\right) s$, see Fig. 2 .

Inflationary models with a large number of e-folds $N_{e}$, will not form PBHs at times earlier than $t_{*}$. But even after $t_{*}\left(N_{e}, \eta\right)$, the difference between the total entropy (given by the GSL) and the Holographic Bound will allow a tiny abundance of PBHs if at all (see Fig. 2). This may be alternatively stated in terms of a threshold redshift $z_{*}$ quenching the formation of PBHs.

\section{Acknowledgments}

Both authors wish to thank the São Paulo State Agency FAPESP for financial support through grants and fellowships. J.E.H. has been partially supported by $\mathrm{CNPq}$ (Brazil). We would like to thank the scientific advice from E. Abdalla on several aspects of this work, and the kind assistance of the Organizers of the Workshop "100 Years of Relativity".
[1] S.W.Hawking, Comm.Math.Phys.43, 199 (1975).

[2] B.Carr, Astron. and Astrophys. Transactions 5, 43 (1994).

[3] A. R. Liddle and A. M. Green, Phys. Reports307, 125 (1998);
A.M. Green and A.R. Liddle Phys. Rev. D60, 063509 (1999). [4] P.S.Custodio and J.E.Horvath, Phys. Rev. D58, 023504 (1998).

[5] B.Carr, Phys. Reports 307, 141 (1998). 
[6] E.W. Kolb and M.S. Turner, The Early Universe, (AddisonWesley, Reading MA 1990)

[7] T. Harada and B.J. Carr, Phys. Rev. D71, 104010 (2005).

[8] Ya. B. Zel'dovich and I.D. Novikov, Relativistic Astrophysics (Univ. Chicago Press, Chicago 1983).

[9] N. G. Sánchez, Phys. Rev. D18, 1030 (1978).

[10] R. Bean and J. Magueijo, Phys. Rev. D66, 063505 (2002).

[11] P.S.Custodio and J.E.Horvath, Int. Jour. Mod. Phys. D14, 257
(2005)

[12] T. Harada and B.J. Carr, Phys. Rev. D72, 044021 (2005).

[13] P.S.Custodio and J.E.Horvath, Phys. Rev. D65, 024023 (2002).

[14] R. Bousso, Rev. Mod. Phys.74, 825 (2002).

[15] D.Bigatti and L.Susskind, TASI lectures on the Holographic Principle, hep-th/0002044 (2000).

[16] J.Bekenstein, Phys. Rev. D9, 3292 (1974). 